\title{
Improving Image Resolution by 45-Degree Tilting-Mode Sampling
}

\author{
Jing $\mathrm{Yu}$ \\ College of Computer Science and Technology, \\ Beijing University of Technology, Beijing 100124, China \\ jing.yu@bjut.edu.cn \\ Zhen-Chun Chang \\ Department of Electronic Engineering, Tsinghua University, \\ Beijing 100084, China \\ txchangchun@163.com
}

\begin{abstract}
A pushbroom sensor consists of a line of sensors arranged perpendicular to the flight direction of the spacecraft. The earth's surface is imaged as the spacecraft flies forward. Supermode sampling using a pair of linear arrays in quincunx arrangement has been implemented on the SPOT5 earth observation satellite. In this paper, we propose a 45-degree tilting-mode sampling model with a square grid, whose sampling density achieves 2 times that of standard sampling, and then apply the estimated MTF of the real tilting-mode imaging system to ORC-based image restoration thanks to the anisotropic spectral support of 45 -degree tilting-mode sampled images. Experimental results on simulated and real images validate the performance of the proposed method. ${ }^{1}$
\end{abstract}

Keywords-Tilting-mode; subpixel sampling; reciprocal cell ; pushbroom.

\section{INTRODUCTION}

Pushbroom sensors are commonly used in satellite cameras for the generation of 2-D images of the earth's surface. In general terms, a pushbroom camera consists of an optical system projecting an image onto a linear array of sensors, typically a CCD array. The pushbroom sensor is mounted on a moving platform, and as the platform moves, the view plane sweeps out a region of space. At regular intervals of time 1-dimensional images of the view plane are captured. The ensemble of these one-dimensional images constitutes a two-dimensional image [1]. In a standard way as shown in Fig.1, successive lines are generated by the satellite motion perpendicular to the linear CCD array. This yields an orthogonal sampling grid, the sampling interval in line and column of which are equal.

In remote sensing field, the high-resolution image is always preferable since it can offer more details for target detection, identification and so on. Several mechanisms have been proposed by the French Spatial Agency (CNES),

\footnotetext{
1 This work was supported by National Natural Science Foundation (No.61501008) and the Open Research Fund of State Key Laboratory of Space-Ground Integrated Information Technology (No.2014 CXJJ-YG 04) of China.
}

\author{
Le-Le Yao \\ Space Star Technology Co. Ltd., State Key Lab. of Space- \\ Ground Integrated Information Technology, \\ Beijing 100086, China \\ ylelexian@163.com \\ Wei-Dong Sun \\ Department of Electronic Engineering, Tsinghua University, \\ Beijing 100084, China \\ wdsun@tsinghua.edu.cn
}

which allow to increase the sampling rate without reducing the size of the sensor. On SPOT5 satellite, supermode sampling has been designed to increase the resolution of the high resolution geometric (HRG) instruments fitted with 2 linear arrays. The 2 linear arrays positioned in quincunx are separated by 0.5 pixels in the row and column directions, and then the sampling rate is doubled in both directions. The HRG instruments generate initially a ground resolution of $5 \mathrm{~m}$, and in the supermode of $2.5 \mathrm{~m}$ [2]. Other novel mechanisms for improving the sampling rate involve both square (hypermode) and hexagonal grids [3]. Supermode, hypermode and hexagonal sampling all use a pair of linear arrays with half-pixel shifts in the line direction. Usually they suffer the following problems: 1) The usage of 2 linear arrays increases the complexity of the imaging device; 2) The accurate registration between a pair of linear arrays is intractable; 3) Additional instruments are needed to ensure that the 2 linear arrays are still separated by 0.5 pixels during skew maneuver observation.

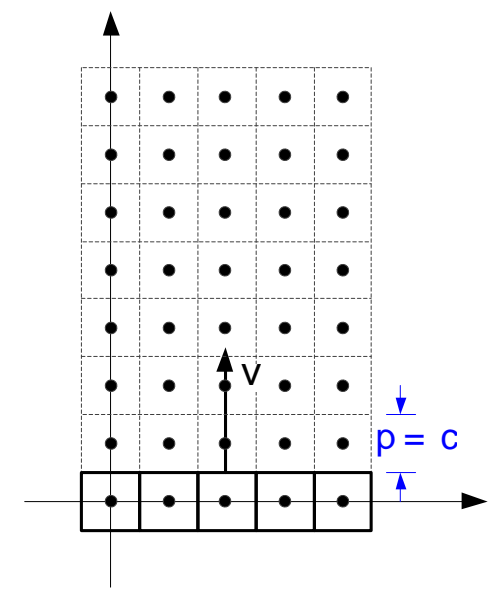

Figure 1. Standard sampling mechanism

The tilting-mode sampling use a typical linear CCD array to improve the resolution by increasing the sampling rate without changing the size of the sensor. During the tilting- 
mode sampling, the linear array of sensors is at an angle, not perpendicular to the moving direction, and simultaneously the sampling period need be controlled accurately along the moving direction to ensure sampling points on a regular grid. In [3], the authors propose an application of the optimal reciprocal cell (ORC) to image restoration for any regular sampling grid. Wang et al. [4] compare the resolution under different sampling systems by simulation experiments. Zhou et al. [5] propose a tilting-mode sampling method, in which the sampling grid forms the shape of a parallelogram, and the image is reconstructed by means of a simple geometric correction. This method has two problems: 1) Sampling density and field of view (FOV) are both limited by the tilting angle; 2) Grayscale interpolation may cause geometric distortion.

In this paper, we present a novel tilting-mode imaging method combining sampling mode and data processing. There are two main contributions of this paper. First, we deduce two conditions the tilting angle and the sampling period of the tilting-mode sampling should satisfy in order to ensure sampling points on a regular square grid. Second, we propose an optimal 45-degree tilting-mode sampling model considering SNR, FOV and sampling density simultaneously, and motivated by spectral characteristics of real 45-degree tilting-mode sampled images, we use ORC for image restoration by the estimation of the anisotropic modulation transfer function (MTF).

\section{OVERVIEW OF SUBPIXEL SAMPLING}

The success of supermode used on SPOT5 satellite, gives a new way to improve the resolution of satellite images. Since then, a series of subpixel sampling schemes have been developed. Figs.2 explain supermode, hypermode and tilting-mode sampling mechanisms, here $c$ is the size of sensors on the linear array, and $p$ is the size of pixels in the image. Supermode and hypermode sampling both use a pair of linear arrays with half-pixel shifts in the line and column directions. The difference between them is that, the sampling period of supermode, corresponding to the integration time, is the same with that of standard sampling, while that of hypermode reduces by half and thus the sampling density is doubled by increasing sampling frequency.

Usually image resolution is defined precisely as the density of the sampling grid:

$$
r_{\text {nom }}=1 / 2(i)=j \operatorname{det}(i) j^{i} 1
$$

Note that $r_{\text {nom }}$ is measured in terms of sampling points per unit area, and image resolution is measured in terms of c.

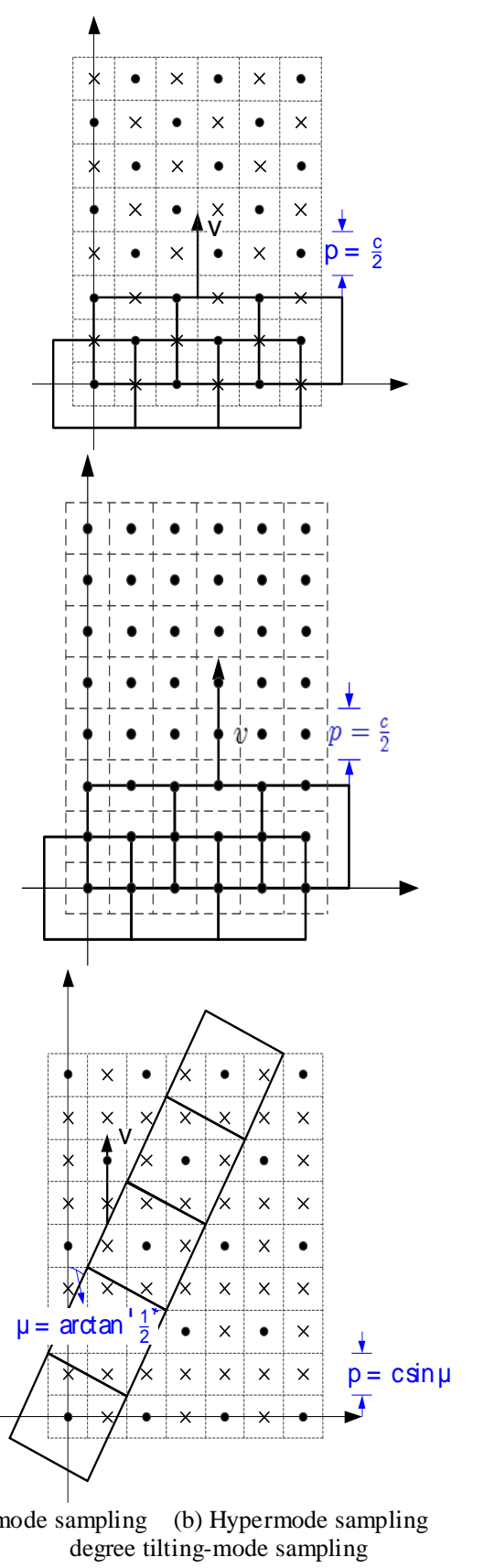

$\begin{array}{lll}\text { (a) Supermode sampling } & \text { (b) Hypermode sampling } & \text { (c) 27- }\end{array}$

Figure 2. Several subpixel sampling mechanisms

Discrete images are assumed to be defined on a regular sampling grid,

$$
\mathrm{i}:=f \mathrm{n}_{1} e_{1}+\mathrm{n}_{2} e_{2} \mathrm{~g}_{\mathrm{n}_{1} ; \mathrm{n}_{2} 2 \mathrm{z}}=Z e_{1}+Z e_{2}
$$

where $f e_{1} ; e_{2} g$ is a basis of $R^{2}$. The standard sampling has nominal resolution $r_{n o m}=c^{i 2}$, where the basis vectors are $(c ; 0)^{\top}$ and $(0 ; c)^{\top}$. In this case, the size of the sensor totally determines image resolution.

Supermode sampling has nominal resolution $r_{\text {nom }}=2 c^{i}{ }^{2}$, where the basis vectors are $(c ; 0)^{\top}$ and $\frac{c}{2} ; \frac{c}{2}{ }^{\top}$; hypermode sampling has nominal resolution $r_{\text {nom }}=4 c^{i}$, 
where the basis vectors are $\frac{c}{2} ; 0^{\top}$ and $0 ; \frac{c}{2}^{\top}$. The sampling density of supermodel and hypermode sampling is 2 and 4 times that of standard sampling, respectively.

27-degree tilting-mode sampling method proposed in [6] has nominal resolution $r_{\text {nom }}=1: 25 \mathrm{c}^{i}$, whose sampling density is 1.25 times that of standard sampling, where the basis vectors are $(4 c \sin \mu ; 0)^{\top}$ and $(2 c \sin \mu ; c \sin \mu)^{\top}$. Although increasing less sampling density than supermode and hypermode sampling, the tilting-mode sampling only use a single linear array, avoiding the difficulties of manufacturing capability and registration accuracy that a pair of CCD lines need solve.

\section{The Proposed Method}

\section{A. Tilting-model Sampling}

Let c denote the size of the sensor, in order to ensure sampling points on a regular square grid, the tilting angle $\mu$ should satisfy,

$$
\begin{aligned}
& \mu=\arctan \frac{1}{n} ; 0<\mu \cdot 1 / 4=4 \\
& \mu=\arctan (n) ; 1 / 4<4<\mu<1 / 4=2
\end{aligned} \quad \mathrm{n}=1 ; 2 ; \mathbb{\$} \mathbb{}
$$

According to the above equation, the tilting angle is $\mu=45^{ \pm}$when $n=1 ; \mu 1 / 427^{ \pm}$or $\mu^{1 / 4} 63^{ \pm}$when $n=2$; $\mu^{1 / 4} 18^{ \pm}$or $\mu^{1 / 4} 72^{ \pm}$when $n=3$, and so on. In this case we do not require extra geometric correction and grayscale interpolation. Obviously, the smaller $\mu$ is, the narrower FOV gets.

The sampling interval between successive lines along the moving direction $\mathrm{d}$ should satisfy,

$$
\begin{aligned}
& d=m c \sin \mu ; 0<\mu . \quad 1 / 4=4 \\
& \mathrm{~d}=\mathrm{mc} \cos \mu ; 1 / 4=4<\mu<1 / 4=2 \quad \mathrm{~m}=1 ; 2 ; \Phi 屯 c
\end{aligned}
$$

As the sampling interval $d$ decreases, the sampling density increases, but the integration time is shortened, resulting in lower signal to noise ratio (SNR) during image acquisition of sensors and bad quality images.

Considering sampling density, SNR, and FOV simultaneously, we propose an optimal 45-degree tiltingmode sampling model, as shown in Fig.3. Different from the above-mentioned 27-degree tiling-mode sampling forming an hexagonal grid, in the case of the 45-degree tilting-mode sampling, discrete images are sampled on a square grid and thus can directly be shown on various general digital image display devices.

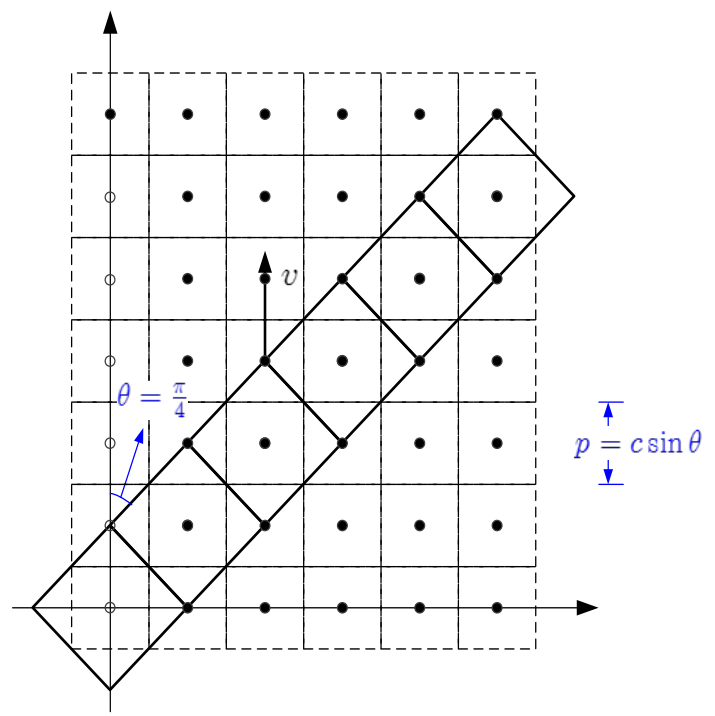

Figure 3. 45-degree tilting-mode sampling mechanism

The nominal resolution $r_{\text {nom }}$ of the tilting-mode sampling at an angle of $\mu=45^{ \pm}$with respect to the vertical is,

$$
r_{\text {nom }}=\operatorname{jdet}\left(e_{1} ; e_{2}\right) j^{i 1}=\operatorname{det} \begin{array}{ccc}
c \sin \mu & c \sin \mu & i 1 \\
0 & c \sin \mu
\end{array}=2 c^{i 2}
$$

where basis vectors of the sampling grid are $\mathrm{e}_{1}=(c \sin \mu ; 0)^{\top}, \mathrm{e}_{2}=(c \sin \mu ; c \sin \mu)^{\top}$.

\section{B. 3.2 Restoration}

In general, the whole process of an image sampling system can be modeled by the following formula:

$$
g(x)=\Phi_{i}(x) \Phi\left(\quad{ }^{i}{ }^{1}(H(u)) \text { a } f(x)\right)+{ }^{\prime}(x)
$$

the imaging system; ' $(x)$ represents the noise due to the imprecision in the sensor measurements; $\Phi_{i}(x)$ represents the geometry of the array of sensors, which are assumed to be distributed on a regular grid; $H(u)$ represents the MTF of the imaging system, which can be viewed as a combination of an optical system, a set of sensors distributed on the focal plane and the motion blur caused by the movement of the linear array during the integration time; $\quad{ }^{1} 1$ represents the inverse Fourier transform.

The spectrum of the sampled image $G(u)$ may contain at different points very different amounts of noise and aliasing. According to equation (6), the Fourier transform of the acquired image can be expressed as a convolution [3]:

$$
\begin{aligned}
G(u) & =f \Phi_{i}(x) g a[H F(u)]+N(u) \\
& =j D j \Phi_{i}{ }^{a}(u) a[H F(u)]+N(u)
\end{aligned}
$$

we can rewrite it in terms of $G_{p u r e}(u)=j D j H F(u)$,

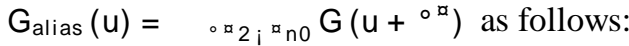

$$
G(u)=G_{\text {pure }}(u)+G_{\text {alias }}(u)+N(u)
$$


where $G(u), F(u)$ and $N(u)$ are the Fourier transforms of $g(x), f(x)$ and $n(x)$, respectively; jDj denotes the area of any tile $D$ of $i$, det $(i)=j D j$. This last expression shows clearly the aliasing and noise errors present in the sampled image $\mathrm{g}(\mathrm{x})$. Thus, it is not appropriate to consider all pixels equal. The relative noise and aliasing are defined as follows:

$$
a(u)=\frac{G_{\text {alias }}(u)}{G_{\text {pure }}(u)}, n(u)=\frac{N(u)}{G_{\text {pure }}(u)}
$$

And then, the optimal reciprocal cell is defined as follows:

$$
\mathrm{D}_{\text {opt }}:=\mathrm{fu}: \mathrm{a}(\mathrm{u})<\mu_{\text {alias }} \text { and } \mathrm{n}(\mathrm{u})<\mu_{\text {noise }} \mathrm{g}
$$

where $\mu_{\text {alias }}$ and $\mu_{\text {noise }}$ are specified thresholds. Observe that equation(10) eliminates the frequency components of the spectrum whenever either the relative aliasing or noise exceeds the specified thresholds.

Now, we use the optimal reciprocal cell for image restoration. We compute the discrete Fourier transform $G(u)$ of the acquired discrete image $\mathrm{g}(\mathrm{x})$. In order to remove aliasing from the image without losing any useful information, we need to upsample the image. We do so either by adding zeros between the samples $\mathrm{g}(\mathrm{x})$ before the Fourier transform, or equivalently by periodically extending $\mathrm{G}(\mathrm{u})$. Then we multiply this periodic $\mathrm{G}(\mathrm{u})$ by $\mathrm{D}_{\mathrm{opt}}$ and take the inverse Fourier transform to obtain an enlarged version of $g(x)$ with some aliased or noisy coefficients removed and some aliased coefficients replaced at their correct locations [3].

A good approach is to apply the Total Variation (TV) regularization on the optimal reciprocal cell $D_{\text {opt }}$. The TV regularization seeks to minimize the total variation

jr $f(x) j d x$. The most important property of the total variation is that in contrast to the Tikhonov regularization the TV allows jumps in the reconstructed signal. The TV regularization is carried out by minimizing the following function,

$$
f^{\wedge}(x)=\arg \min _{f}^{-} j r f(x) j d x+{ }_{x 2 i}^{x} j(h \circ f)(x) i \quad g(x) j^{2}(11)
$$

where , is a properly chosen Lagrange multiplier.

According to Parseval's theorem, the data-fitting term can equivalently be rewritten in the Fourier domain as follows,

$$
f^{\wedge}(x)=\arg \min _{f}{ }^{-} j r f(x) j d x+, \quad D_{\text {vor }} j H F(u) i \quad G(u) j^{2} d u(1
$$

We integrate the data-fitting term over the $D_{\text {opt }}$ (after periodic extension of the Fourier spectrum) instead of over the $\mathrm{D}_{\text {vor }}[3]$,

$$
f^{\prime}(x)=\arg \min _{f}{ }^{-} j r f(x) j d x+,{ }_{D_{\text {opt }}} j H F(u) \text { i } G(u) j^{2} d u(13)
$$

The benefit of doing so becomes evident when we compare the solutions of equations (12) and (13) as shown in Fig.5(b) and (d), respectively.

\section{EXPERIMENTAL RESULTS}

\section{A. Experiments with Simulated Images}

The spectral support of the tilting-mode sampled image is anisotropically elliptic with the major axis along the line direction. If the tilting-mode sampling system has a heavily anisotropic MTF, then it is easier to correct aliased Fourier coefficients of sampled images, and the advantages of the optimal reciprocal cell becomes more evident. In this experiment, we simulate the MTF of the 45-degree tiltingmode sampling system. Fig.4(a) (d) depict the original image, the level-lines of the simulated MTF, and the degraded image with a signal-to-noise ratio of $48 \mathrm{~dB}$, respectively. As we can be seen from Fig.4(b), the MTF is strongly anisotropic.

Then the reciprocal cell is applied to TV regularization in the Fourier domain. Figs.5(a) and (b) show the Voronoi reciprocal cell $D_{\text {vor }}$ and the reconstructed result with TV regularization over $D_{\text {vor }}$; Figs.5(c) and (d) show the optimal reciprocal cell $D_{\text {opt }}$ and the reconstructed image with TV regularization over $D_{\text {opt }}$, here thresholds $\mu_{\text {alias }}$ and $\mu_{\text {noise }}$ are set to 0.2 and 5 . The reconstruction quality depends highly on the anisotropic MTF. The optimal reciprocal cell can not only remove aliasing and noise, but also take full advantage of the high-frequency Fourier coefficients outside the Voronoi cell by translating the Fourier coefficients to their correct locations.

where $f(x)$ represents the ideal image just before it enters

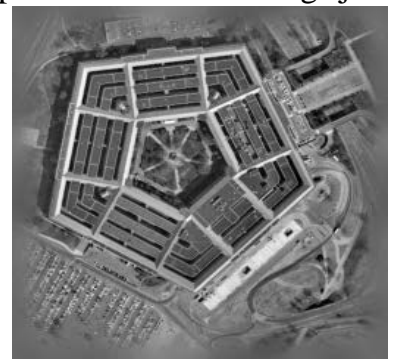

(a)Original image 


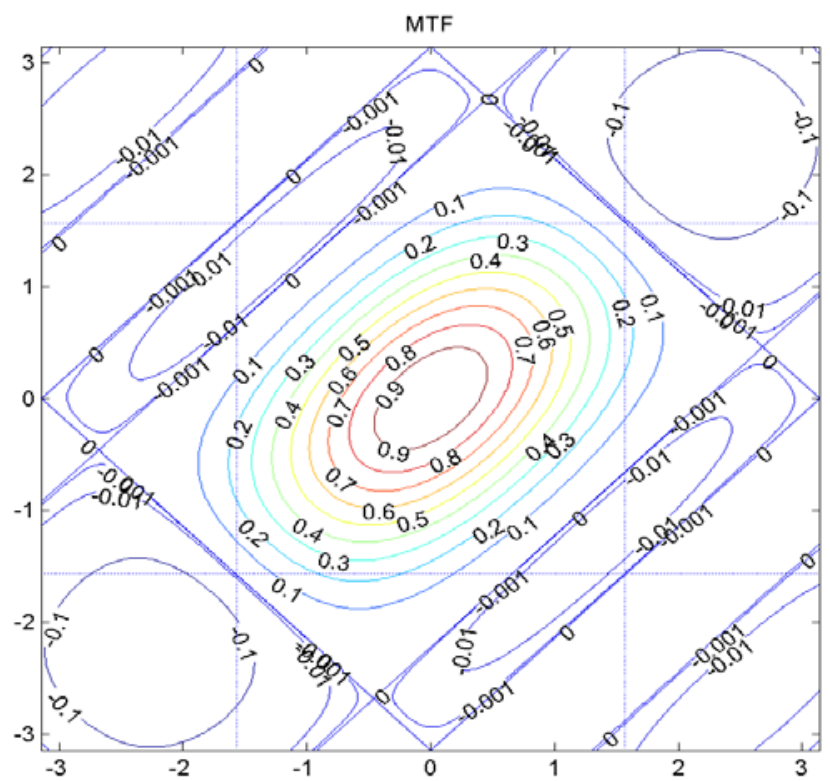

(b) MTF of simulated tilting-mode system

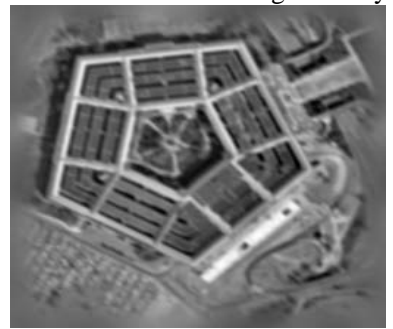

(c) Degraded image

Figure 4. 45-degree tilting-model sampling simulation

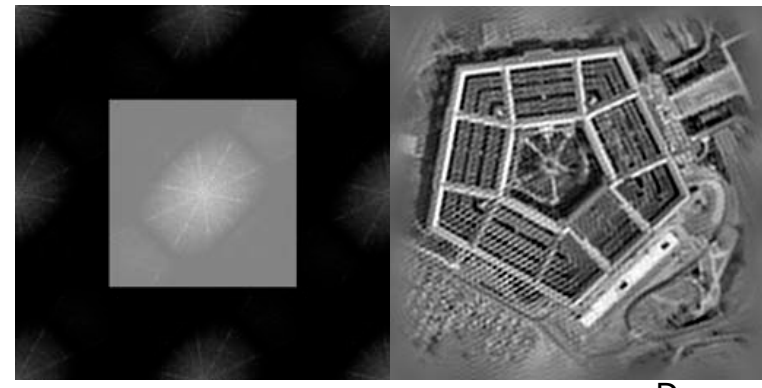

(a) Voronoi reciprocal cell (b) Deconvolved by TV over $D_{\text {vor }}$
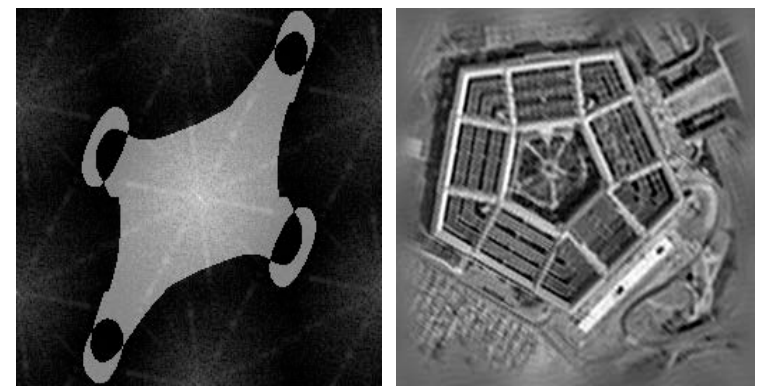

(c) Optimal reciprocal cell (d) Deconvolved by TV over $D_{\text {opt }}$

Figure 5. Comparison between the deconvolution over $D_{\text {vor }}$ and $D_{\text {opt }}$ for the simulated images

\section{B. Experiments with Real Images}

On our tilting-mode image acquisition platform, the pushbroom CCD camera moves on the sliding screw at a uniform speed with a fixed tilting angle. The camera parameters are as follows: the sampling rate is $33 \mathrm{fps}$, the focal length is $23 \mathrm{~mm}$, and the size of the sensor is 0.0074 $\mathrm{mm}$. During capture the actual object distance is $4 \mathrm{~m}$ and accordingly the moving speed is $30 \mathrm{~mm} / \mathrm{s}$ so as to ensure sampling points on a regular square grid. Fig. 6 shows a real 45-degree tilting-mode sampled image of the resolution chart. Because of the cut-off frequency of the optical lens and the sensor of the acquisition system, the sampling rate, camera movement during the exposure and the other factors, the captured image tends to blurring. We try to deconvolve the whole MTF including the sensor, the optics and the motion blur.

The accurate estimation of the MTF is the key to success of image restoration. In this paper, the blur kernel estimation method in [7] is used to estimate the MTF, and the size of the estimated blur kernel is $23 £ 25$. Fig.7(a) shows the level-lines of the estimated MTF, in which the dashed lines represent the boundaries of the Voronoi reciprocal cell. Clearly there are more high-frequency components along the linear array in the Fourier spectrum of the real 45-degree tilting-mode sampled image, and therefore, the spectral support is elliptic with the major axis at an angle of 45 degrees from the horizontal. Fig.7(b) shows the ORC for the 45-degree tilting-mode sampled image. Fig.7(c) shows the result of bilinear interpolation method with an upscaling factor of 2, and Fig.7(d) shows the reconstructed image of the proposed method. Experimental results show that our method can remove motion blur, and make the real tilting-mode sampled image become clear.

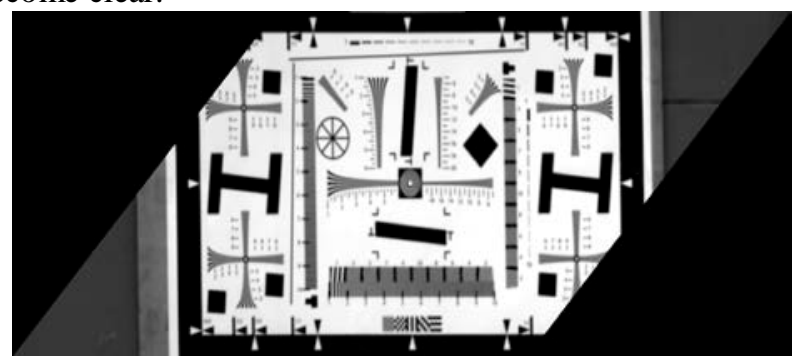

Figure 6. Real 45-degree tilting-mode sampled image 


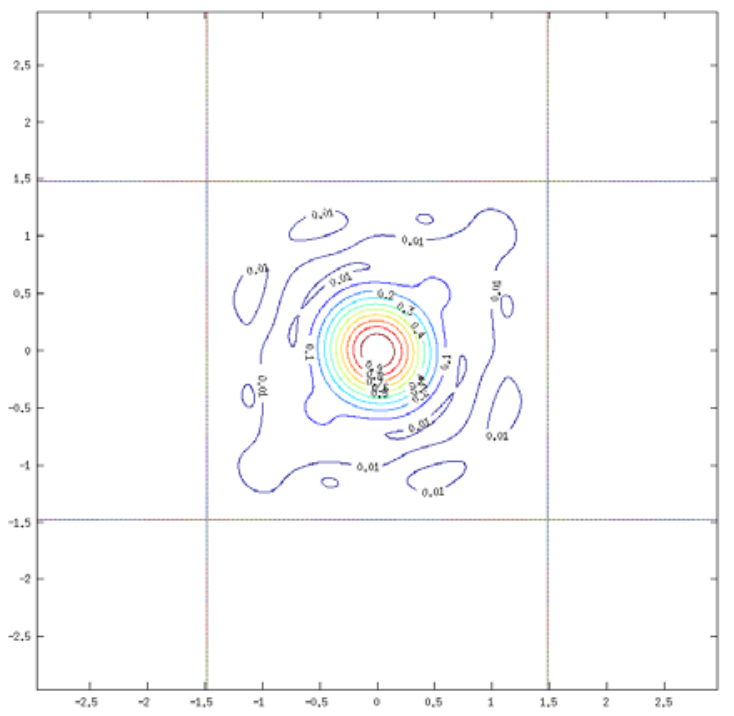

(a) MTF estimation

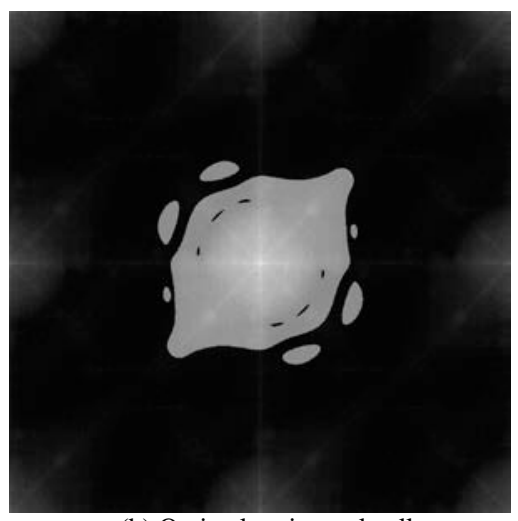

(b) Optimal reciprocal cell

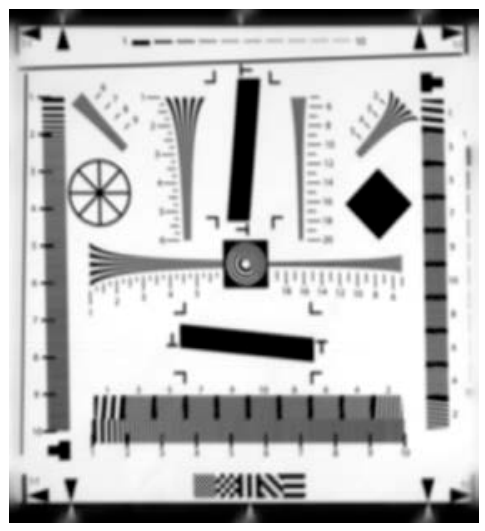

(c) Bilinear interpolation

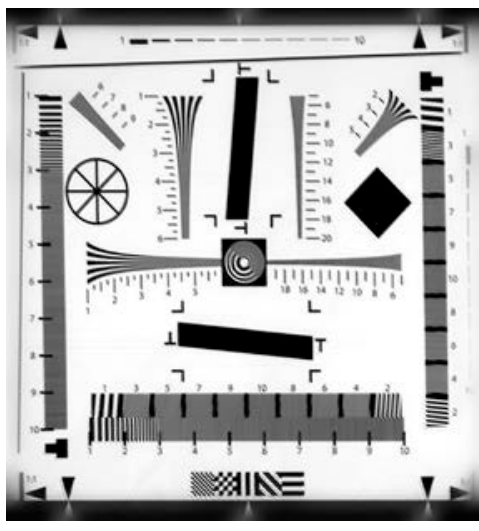

(d) Our proposed method

Figure 7. MTF and reconstructed result of the real tilting-mode sampled image

\section{CONCLUSION}

Here, we proposed a novel 45-degree tilting-mode sampling method. In this method, the optimal reciprocal cell combined with the MTF estimation is used to restore tiltingmode sampled images. Experimental results show that our proposed method can improves image resolution compared with the standard sampling, and removes aliasing and blurring though the kernel estimation of the real tiltingmode sampling system, due to the unique anisotropic characteristics of its MTF.

\section{REFERENCES}

[1] 1. R. I. Hartley and R. Gupta, "Linear pushbroom cameras," in Third European Conference on Computer Vision, Stockholm, Sweden, 1994, pp. 555-566.

[2] 2. P. Goudy, P. Kubik, B. Rouge, and C. Latry, "From image processing concepts to instrument design in remote sensing satellites,", 2002, pp. 431-434.

[3] 3. A. Almansa, S. Durand and B. Rougé, "Measuring and improving image resolution by adaptation of the reciprocal cell," Journal Mathematical Imaging and Vision, vol. 21, pp. 235-279, 2004.

[4] 4. J. Wang, L. Y. Xu and D. S. Xia, "The aliasing analysis and resolution calculation of tilting sampling system," ACTA ELECTRONICA SINICA, vol. 40, pp. 1067-1072, 2012. (in Chinese)

[5] 5. F. Zhou, H. Wang and W. Ma, "A study on a new method for improving image spatial resolution of sampled optical imager with single array," Journal of Astronautics, vol. 27, pp. 227-232, 2006. (in Chinese)

[6] 6. Y. Zheng, Y. Tang and Q. Chen, "A method for increasing effective resolution of tilting mode satellite image," Journal of Computer-Aided Design \& Computer Graphics, vol. 21, pp. 243-249, 2009. (in Chinese)

[7] 7. L. Xu and J. Jia, "Two-phase kernel estimation for robust motion deblurring," in European Conference on Computer Vision, Heraklion, Greece, 2010, pp. 157-1 